\title{
Fachsprachliche Bildung in der Geographie
}

"sonja.schwarze@uni-muenster.de, Institut für Didaktik der Geographie, Universität Münster

Obwohl Sprache im Fach Geographie in den vergangenen Jahren eine neue Relevanz erfahren hat, liegen für das junge Forschungsfeld der Sprachbildung im Fachunterricht zum jetzigen Zeitpunkt noch wenige empirische Ansätze vor. In diesem Artikel werden daher insbesondere aus der Theorie abgeleitete Handlungsempfehlungen aus dem modernen Fremdsprachenunterricht, dem bilingualen Sachfachunterricht und dem Deutsch-als-Zweitsprache-Unterricht vorgestellt, die für einen sprachsensiblen Geographieunterricht fruchtbar gemacht werden können. Die Handlungsempfehlungen werden mit Blick auf den Umgang mit einem diskontinuierlichen Text in einem Scaffolding als Umsetzungsbeispiel für fachlich und sprachlich integriertes Lernen visualisiert.

Keywords: (Fach-)Sprachbildung im Fachunterricht, theoriebasierte Handlungsempfehlung, Scaffolding für diskontinuierlichen Text

\section{Educating technical language of the content subject Geography}

Although language has been experiencing a new relevance in Geography for the last years now, there are still only few empirical approaches available in the young research field of language sensitive teaching in content subjects. Therefore, this article presents theory-based recommendations derived from modern foreign language teaching, Content and Language Integrated Teaching (CLIL) and teaching German as a second language that can be used as fertile resources to make Geography more sensitive towards students' language challenges. The recommendations are visualized with the aid of a scaffolding focusing on a discontinuous text as an example of integrative content and language teaching.

Keywords: language sensitive teaching, theory-based recommendations, scaffolding for discontinuous text

\section{Die Relevanz der Sprache in Lernprozessen}

Sprache ist ein wesentlicher Bestandteil jeden Lernens (vgl. Schmölzer-Eibinger et al. 2012: 11), der in der Fachdidaktik jedoch erst in den vergangenen Jahren an Bedeutung gewonnen hat (vgl. SchroeterBrauss 2013: 127). Ohne sprachlichen Zugang und geeignete kommunikative Mittel zur eigenständigen Aneignung und sprachlichen Aushandlung von Lerngegenständen und Sachverhalten, wird Schülerinnen und Schülern die Teilhabe am Unterricht, an der Bildung und letztlich an der Gesellschaft verwehrt. „Sprachliche Bildung und Förderung müssen als Teilaspekt der Ermöglichung von Chancengleichheit und gesellschaftlicher Teilhabe gesehen werden" (Jeuk 2013: 102). Ein Unterricht, der sowohl auf fachliche als auch auf sprachliche Bildung abzielt, berücksichtigt den Sprachstand der Lernenden und „holt sie an der Stelle ab, an der sie momentan stehen" (Beese at al. 2015: 7). Im Fokus stehen dabei insbesondere zwei Schüler/innengruppen: Lernende, die einen Migrationshintergrund aufweisen sowie sprachschwache Schüler/innen im Generellen.

Die Schüler/innenschaft in der Bundesrepublik Deutschland zeichnet sich durch einen hohen Migrationsanteil aus, der bundesweit bereits bei über 20\% liegt und stetig steigt (vgl. Leisen 2013: 18 zit. n. Konsortium Bildungsberichterstattung 2006: 142). Bei vielen Schülerinnen und Schülern stellt Deutsch entsprechend die zweite Sprache dar, die sie zum Teil erst mit Eintritt in den Kindergarten oder gar in die Grundschule erwerben (vgl. Jeuk 2013: 14). Oftmals täuschen eine nahezu fehlerfreie Aussprache und hohe alltagssprachliche Kompetenzen darüber hinweg, dass sich die Schüler/innen noch im Spracherwerbsprozess befinden. Insbesondere die Überführung von der All- 
tagssprache in die Bildungs- sowie Fachsprache(n), bei denen es sich um konzeptionell schriftsprachliche Kommunikationsverwendungen handelt, bereitet ihnen große Mühen (vgl. Kniffka \& Siebert-Ott 2012: 22; vgl. Jeuk 2013: 53, 73 f.). Der Erwerb der schriftlichen Sprachvariante bei Schülerinnen und Schülern mit Deutsch als Zweitsprache (L2) folgt einem anderen Verlauf als bei Schülerinnen und Schülern, deren Erstsprache (L1) Deutsch ist. Durch den zeitlich versetzten Spracherwerb können sich heterogene Sprachstände in den L1- und L2-Schüler/innenkohorten ergeben. Ohne passenden Erwerb und Förderung der Schriftsprache werden die Absolvierung höherer Bildungswege und damit einhergehend höherer beruflicher Qualifizierungen erschwert sowie letztlich gesellschaftliche Teilhabe verwehrt (vgl. Röhner 2013: 9; vgl. Rösch 2013: 19; vgl. Hövelbrinks 2013: 76). Die Beherrschung von Sprache bzw. ihre Ermangelung wirkt als machtvolles Werkzeug zur Inklusion bzw. Exklusion und weist somit auf die sozialsymbolische Funktion von Sprache hin (vgl. Oleschko et al. 2017: 11).

$\mathrm{Zu}$ bedenken ist ebenfalls, dass sich auch L1-Schüler/innen noch im Spracherwerbsprozess befinden und zwar mit Blick auf (deutsche) Bildungs- und Fachsprachen. Ebenso charakterisieren sie sich oftmals durch heterogene rezeptive und produktive Sprachfähigkeiten in ihrer Erstsprache; ein Phänomen, das speziell beim fachlichen Lesen und Schreiben im Unterricht beobachtet werden kann. Die sich noch ausbildenden sprachlichen Fähigkeiten auf den individuell unterschiedlichen Niveaus können sich daher auch für L1-Lernende zur Hürde beim Lernen entwickeln. Sprachstarke Schüler/innen erwerben fachsprachliche Strukturen unbewusst nebenbei und nutzen diese zielführend für fachliche Aushandlungen, während sprachschwächere Schüler/innen zunächst eine bewusste und systematische Bildung oder Förderung von Fachsprache benötigen.

Spätestens seit den Ergebnissen der PISA-Studien $\mathrm{zu}$ sprachlichen Bereichen im Lernen und insbesondere den Ergebnissen von L2-Schülerinnen und Schülern wird Sprache in der Bildung differenzierter adressiert (vgl. Röhner 2013: 7 f.). Damit ist die Einsicht verbunden, die Rolle der Sprache und Kommunikation als Voraussetzungen für Lernprozesse im institutionellen Kontext neu zu evaluieren und geeignete Handlungsoptionen im fächerübergreifenden sowie fachspezifischen Unterricht zu entwickeln. Denn schließlich stehen fachliches und sprachliches Lernen in einem engen reziproken Verhältnis und lassen sich nicht isoliert erlernen (vgl. Grießhaber 2013: 58f.). „Lernen heißt - in welchem Fach auch immer - eine Aneignung von Sprache" (Ahrenholz 2010: 17). Die Anbahnung sprachlicher Kompetenzen und Textrezeption sowie -produktion (Texterschließung, Lesen und Schreiben) (vgl. Rösch 2013: 21) fällt deshalb in den fachspezifischen Verantwortungsbereich jeder Lehrerin bzw. jeden Lehrers. Alle Schüler/innen, ob mit oder ohne Zweitsprachenhintergrund, sollten daher fach- und bildungssprachlich gefördert werden, da sich die Sprache des Unterrichts und der Bildung erheblich von der Alltagssprache unterscheidet.

Ferner muss an dieser Stelle explizit angesprochen werden, dass ein Migrationshintergrund und die damit verbundene Mehrsprachigkeit der Lernenden nicht zwangsläufig sprachliche ,Defizite' oder ,Schwierigkeiten' von Schüler/innen bedingen. Die persistente negative Konnotation, die mit Mehrsprachigkeit und Migrationshintergrund verbunden wird, gilt es bewusst aufzubrechen. Studien weisen daraufhin, dass nicht der Migrationshintergrund und die Mehrsprachigkeit grundsätzlich zu Bildungsbenachteiligung führen, sondern diese eher mit der sozialen Schicht einhergehen (vgl. Jeuk 2013: 20). Der Migrationshintergrund bzw. die Mehrsprachigkeit können gegebenenfalls eine Herausforderung für den bildungssprachlichen Erwerb darstellen, von Kausalität kann aber keinesfalls gesprochen werden. Viele Schüler/ innen, die Deutsch als L2 erwerben, weisen hervorragende alltags- und fachsprachliche Kompetenzen auf. Mehrsprachigkeit verkörpert des Weiteren eine immense sprachliche wie auch kulturelle Ressource und Potential. Mit Blick auf das Postulat der KMK und der Europäischen Union, eine mehrsprachige, globalisierte Schüler/innen- und Gesellschaft zu fördern, in der die Mitglieder in bis zu drei Sprachen kompetent agieren können (vgl. KMK 2013: 5 ff.), wird entsprechend eine neue Bewertung von Mehrsprachigkeit evident ${ }^{1}$.

\section{Sprachsensibler Geographieunterricht}

\subsection{Sprachbewusstheit im Geographieunterricht}

In den vergangenen Jahren trat ein verstärkt sprachsensibel durchgeführter Geographieunterricht in Erscheinung, der der Sprache in der Unterrichtsvor-

\footnotetext{
Bislang wurden der Migrationshintergrund und die damit assoziierte Mehrsprachigkeit von Schüler/innen mit einer anderen Erstsprache als den Prestigesprachen Englisch, Französisch, Spanisch und gegebenenfalls den skandinavischen Sprachen als defizitär und problematisch betrachtet. Diese negative Konnotation wird im Zuge der aktuellen Entwicklungen bewusst aufgebrochen (vgl. Jeuk 2013: 11). Denn schließlich stellen alle Sprachen eine Ressource dar (vgl. ebd. 2013: 7, 23, 105). Theorien gehen ferner davon aus, dass je stärker ein/e Lernende/r bildungssprachliche Strukturen in der Erstsprache erworben hat, desto schneller und müheloser erwirbt die/der Lernende diese Strukturen in der Zweitsprache Deutsch (vgl. Kniffka \& Siebert-Ott 2012: 181; vgl. Jeuk 2013: 50). Mehrsprachigkeit kann daher eine klare Lernchance darstellen.
} 
bereitung und -durchführung eine veränderte, bewusstere Rolle einräumt. Erste Ansätze einer bewussteren Adressierung von Sprache in der Geographie ließen sich bereits in der Etablierung des Kompetenzbereichs ,Kommunikation' in den Bildungsstandards (vgl. DGfG 2014: 21 ff.) identifizieren. Die Ansätze gilt es nun konkret auf verschiedenen sprachlichen Ebenen (von der Wort- bis Textebene) zu präzisieren und gezielt Unterrichtsmaßnahmen zur integrierten Sprachbildung umzusetzen.

Geographieunterricht sprachsensibel durchzuführen bedeutet nicht, dass Lehrkräfte die relevante Fachsprache und sämtliche Materialien komplett vereinfachen sollen. Ein solch defensives Vorgehen wird der Komplexität des Faches nicht gerecht. Es werden Fachtermini /-phrasen, komplexe Satz- sowie Textstrukturen gebraucht, um geographische Sachverhalte explizit analysieren und diskutieren zu können. Neben der kommunikativen Funktion von Sprache, rückt bei einem sprachsensiblen Fachunterricht insbesondere die epistemische Funktion (Sprache als Werkzeug zum Denken) in den Fokus (vgl. Oleschko et al. 2017: 14f.). Geographische Denkoperationen und -prozesse benötigen schließlich Fachsprache, da sonst komplexer werdende, abstrakte Sachverhalte nicht präzise erfasst, verknüpft und artikuliert werden können. Um autonom im Erarbeiten geographischer Fragestellungen zu werden, müssen Lernende diese Strukturen erfahren und erlernen können. Das heißt nicht, dass Fachlehrer/innen als Sprachlehrer/ innen fungieren sollen. Es geht eher darum, dass sie ihre Fachperspektive um den Bereich Sprache und Kommunikation erweitern, um geographische Kompetenzen und die für den Lerngegenstand relevante Fachsprache integrativ anzubahnen. Daher betont der sprachsensible Geographieunterricht den behutsamen und bewussten Einsatz von Maßnahmen zur (Fach-) Sprachbildung.

Zunächst müssen Lehrkräfte dafür die im Fach genutzte Sprache bewusst wahrnehmen (vgl. Beese 2013: 39, 51), um mit Blick auf den Lerngegenstand sowie die jeweilige Lerngruppe passende Entscheidungen fällen zu können. Ein sprachsensibler Unterricht beleuchtet also die genuine Beziehung des Faches und der Sprache, denn jedes Fach nutzt bildungssprachlich ähnliche aber auch z.T. ganz fachspezifische Sprachstrukturen. Der Geographieunterricht verwendet bspw. eigene Fachtermini und fachspezifische Redemittel zum Auswerten geographischer Arbeitsmethoden und bedarf grammatikalisch betrachtet sämtlicher Tempusformen zur Untersuchung geographischer Sachverhalte, während bspw. der Chemieunterricht fast ausschließlich das Präsens nutzt.

Noch befindet sich das Forschungs- und Praxisfeld des sprachsensiblen Geographieunterrichts in seinen Anfängen, weshalb bisher kaum auf empirisch abgesicherte Erkenntnisse zurückgegriffen werden kann. Vorläufig können theoretische und empirische Erkenntnisse aus dem bilingualen Sachfach-, dem modernen Fremdsprachen- und Deutsch-alsZweitsprachunterricht zu Rate gezogen und für einen sprachbewussten Geographieunterricht fruchtbar gemacht werden. Empirische Forschungen bezüglich der Effektivität dieser Maßnahmen folgen; ebenso die Erforschung dessen, wie sich die Sprachen der Fächer spezifisch voneinander unterscheiden.

\section{BICS (Alltagssprache) vs. CALP (Fach- und Bildungssprache)}

Um die geographische Fachsprache adäquat im Unterricht anbahnen zu können, sollten sich Lehrer/innen zunächst vergegenwärtigen, wie sich die Alltags- und Fach- also allgemeiner gesprochen die Bildungssprache ${ }^{2}$ voneinander unterscheiden. Jim Cummins differenziert Sprache bereits 1979 in zwei Bereiche: BICS (Basic Interpersonal Communicative Skills) und CALP (Cognitive Academic Language Proficiency) (vgl. Kniffka \& Siebert-Ott 2012: 21 f; vgl. Jeuk 2013: $51 \mathrm{ff}$.$) . BICS bezeichnen grundlegende Kommuni-$ kationsfähigkeiten zur Bewältigung von alltäglichen Kommunikationssituationen, während CALP die kognitiv-akademischen Sprachfähigkeiten, die für das schulische Lernen relevant sind, darstellt. Die Alltagssprache (BICS) zeichnet sich durch eine konzeptionell mündliche Sprachvariante aus, in welcher Sätze oftmals syntaktisch vereinfacht und unvollständig formuliert werden. Viele Füllwörter werden verwendet und Wiederholungen sowie narrative Erzählungen mit unpräzisem Wortgebrauch treten auf. Ferner ist Alltagssprache kontextbezogen und beinhaltet Gestik, Mimik, Körperhaltung und Tonfall als wesentliche Kommunikationsträger. Demgegenüber stehen die Merkmale von Bildungssprache (CALP).Es handelt sich um komplexe, vollständige und syntaktisch korrekte Sätze mit Nebensatzstrukturen, die einen präzisen Fach- und Bildungswortschatz aufweisen. Meist sind sie frei von Redundanz oder Füllmaterial und folgen einer linearen Argumentation. Ferner ist die Kontextfreiheit ein Charakteristikum. Bildungssprache kann entsprechend als konzeptionell schriftliche Sprachvariante bezeichnet werden. Die Bezeichnung ,konzeptionell' verweist darauf, dass diese Sprachvarianten nicht zwangsläufig medial verschriftlicht oder mündlich gesprochen werden, sondern dass sie

\footnotetext{
An dieser Stelle sollte auf Diskussionen und terminologische Unschärfen um die Begriffe hingewiesen werden (vgl. Jeuk 2013; Kniffka \& Siebert-Ott 2012; Schmölzer-Eibinger 2012; Leisen 2013 u.v. m.).
} 
sich durch die aufgeführten Merkmale charakterisieren (vgl. Hövelbrinks 2013: 76). So wird bspw. ein mündlich präsentierter Vorlesungsvortrag der Kategorie der konzeptionell schriftlichen Sprachverwendung zugeordnet, ein geschriebener Chat unter Bekannten jedoch eher der Kategorieder konzeptionell mündlichen Sprachverwendung (vgl. Jeuk 2013: 53).

\subsection{Geographische Fachsprache}

An dieser Stelle wird nun die geographische Fachsprache genauer betrachtet, denn Bildungssprache ist die generalisierte Summe übergeordneter Sprachstrukturen aus verschiedenen Fachsprachen, die sich - selbst trotz vieler Gemeinsamkeiten - unterscheiden und eigene sprachliche Besonderheiten aufweisen (vgl. Rösch 2013: 22; s.a. Hövelbrinks 2013: 77). Ein Blick in die Spezifika der geographischen Fachsprache auf den verschiedenen Sprachebenen, von der Wort- bis zu der Textebene, ist lohnenswert (vgl. Schmölzer-Eibinger et al. 2012: 12). Die bewusste Adressierung der Bereiche Lexik (Wörter/ Wortschatz) und Morphologie (Struktur und Bauformen von Wörtern), Syntax (Bau von Sätzen, vgl. Vollmer 2005: 88, 101, 115) und der im Fach genutzten kontinuierlichen und diskontinuierlichen Textsorten sensibilisiert für eventuelle ,Stolpersteine' (vgl. Rösch 2005) von Schülerinnen und Schülern beim Lernen. Bei Stolpersteinen handelt es sich um Besonderheiten der deutschen Sprache, die Lernenden Schwierigkeiten bereiten können. Ein aufgeklärtes sprachliches Bewusstsein bietet Möglichkeiten im Umgang mit potentiellen Stolpersteinen und einer gezielten Anbahnung fachsprachlich relevanter Strukturen. Für dieses Teilkapitel wurden insbesondere theoretische Überlegungen aus Deutsch-als-Zweitsprach- und modernem Fremdsprachenunterricht zu Rate gezogen.

\section{Lexik}

Auf die anfängliche Frage in Lehrveranstaltungen, was die geographische Fachsprache aus Sicht von Lehramtsstudierenden charakterisiert, wird fast ausschließlich auf den Aspekt Fachtermini eingegangen (s. a. Grießhaber 2013: 58). Bei gezieltem Blick findet man jedoch weitere Spezifizierungen. Im Bereich der Lexik (Wörter) wird eine große Anzahl an Fachtermini genutzt, die für die Geographie charakteristisch, in anderen Fächern aber weniger präsent sind (z. B. Landschaftszone, Bodenfruchtbarkeit, Niederschlagsmaximum). Ferner zeichnet sich die Geographie dadurch aus, dass sie oftmals terminologisierte Alltagssprache verwendet (z. B. Landschaft, Stadt). Die Begriffe sind alltagssprachlich bekannt und werden von den Lernenden häufig benutzt. Im Fach haben sie jedoch eine eindeutige definitorische Bedeutung, die sich vom Alltagsbegriff unterscheidet. Genau hier liegt ein Stolperstein für Lernende.

Des Weiteren werden in der geographischen Fachsprache (wie auch in anderen Fachsprachen) oftmals sogenannte Funktionsverbgefüge mit einem bedeutungsschwachen Verb (z. B. „Entstehung erfolgt" statt „entstehen“; vgl. Schroeter-Brauss 2013: 127) verwendet. Gebildet werden diese aus einem Substantiv und Verb. Das Verb zeigt in diesem Zusammenhang nicht die Tätigkeit oder den Vorgang an, sondern hat eine dienende Funktion in der Kollokation. Dies ist jedoch insbesondere für Lernende des Deutschen eine Schwierigkeit, haben diese doch gelernt, das Verb als Bedeutungsträger zu identifizieren. Das Verb „erfolgen“ wechselt jedoch in Verbindung mit „Entstehung" seine semantische Funktion. Die Erläuterung des Funktionsverbgefüges im Plenum könnte helfen, den möglichen Stolperstein zu beseitigen.

\section{Morphologie}

Ebenfalls mit dem Bereich der Lexik assoziiert ist die Morphologie, welche sich mit der inneren Struktur von Wörtern beschäftigt und Morpheme, „die kleinsten bedeutungstragenden Elemente der Sprache" (Karatas 2005: 87) erforscht. Gemeint ist damit der, Wortaufbau'. Wie alle Fachsprachen nutzt auch die Geographie ein großes Spektrum an (Mehrfach-) Komposita - Wörter, die aus anderen zusammengesetzt werden und daher eine neue Bedeutung erhalten. Als Beispiele seien genannt: Inselbildungsprozess, Tieflandregenwälder, Vulkanausbruch. Die neuen Wörter können sich dabei aus Nomen, Verben und Adjektiven zusammensetzen. Das Prinzip der Wortbildungsprozesse ist ein normaler Bestandteil der Sprache, der sich auch in der Wortderivation wiederfindet. Dabei handelt es sich um die Bildung neuer Wörter durch das Hinzufügen von Prä- oder Suffixen (Vor- oder Nachsilben) bzw. um deren Abtrennung (vgl. Schöneck 2005: 99 ff.). Oftmals wird dadurch die Wortklasse verändert. Zum Beispiel wird aus dem Nomen ,Produktivität' durch Abtrennen des Suffixes das Adjektiv ,produktiv' oder aus dem Nomen, Wirtschaft' durch das Hinzufügen des Suffixes, -lich' das Adjektiv, wirtschaftlich'. Die Wortklasse kann aber auch z.B. durch Hinzufügen des Präfixes ,un-' beibehalten, aber die Bedeutung des Wortes verneint werden: ,unwirtschaftlich'. Ähnlich verhält es sich mit zusammengesetzten und abgeleiteten Adjektiven, die meist mit den Endungen ,-arm', ,-reich', '-bar', ,-los', gebildet werden, wie die Adjektive ,nährstoffarm', ,sauerstoffreich', ,arbeitslos' als Beispiele zeigen. Ein weiteres morphologisches Charakteristikum der Fachsprache ist die Verwendung von Nominalisie- 
rungen, in denen Verben substantiviert werden (z. B. beim Vermessen, während des Untersuchens etc.; vgl. Schroeter-Brauss 2013: 127).

Wie geht man nun im Unterricht geschickt mit diesen lexikalischen und morphologischen Feinheiten um? Verschiedene Handlungsmöglichkeiten, die sich im Deutsch-als-Zweitsprachen-, im modernen Fremdsprachen- und im bilingualen Sachfachunterricht bewährt haben, können angedacht werden. Neue Termini sollten mit Artikel und Pluralform eingeführt und semantisiert werden (Umschreibung, um die Bedeutung von Wörtern zu erfassen), da der Genus (Geschlecht) oft nicht einfach aus dem Wort abgeleitet werden kann und im Deutschen bis zu acht Varianten existieren, um den Plural zu bilden (vgl. Leisen 2013: 184 ff.; vgl. Jeuk 2013: 68 ff.). Die verwendeten Funktionsverbgefüge sollten im Kontext besprochen und bewusst eingeführt werden. Ferner bietet es sich an, ein thematisches Vokabular mit den wesentlichen Fachbegriffen der Unterrichtsreihe anzufertigen, auf welches die Schüler/innen in verschiedenen Kontexten gezielt zurückgreifen können. Dabei bieten sich Hefteinträge und im Klassenraum ausgestellte Lernposter an, die den jeweilig benötigten Fachwortschatz einschleifen, Sachverhalte reaktivieren und von Schülerinnen und Schülern aktiv genutzt werden. Auch Semantisierungstechniken und Übungen zur Festigung des Wortschatzes ähnlich wie in den Fremdsprachen werden empfohlen, die den Wortschatz durch Anregung unterschiedlicher Lernkanäle umwälzen und kognitiv verankern (vgl. Haß 2006: $114 \mathrm{ff}$.; Leisen 2013: 185). Als Beispiele neben vielen weiteren lassen sich das Worträtsel, Lückentexte, Definitionen und Mnemotechniken nennen.

Zwischen der Wort- und Satzebene bietet die Phraseologie interessante Erkenntnisse, bei der ,redensartliche Verbindungen' betrachtet werden (vgl. Vollmer 2004: 24). Oftmals treten Wortfolgen in bestimmten Kontexten als Phrasen gemeinschaftlich auf. Bei der Auswertung von Klimadiagrammen bspw. werden diese Phrasen unabhängig vom jeweiligen Diagramm immer wieder eingesetzt. Gerade diese Phrasen lassen sich als fachspezifische Redemittel transparent mit Schülerinnen und Schülern erarbeiten und einüben. Schließlich sind diese die ersten Bausteine zur Formulierung von Texten im Fach.

Des Weiteren sollten metasprachliche Phasen sinnstiftend eingebaut werden, wenn sich diese aus dem fachlichen Kontext heraus ergeben. Gemeint ist damit ein bewusstes Reflektieren der genutzten Fachsprache. Beispielsweise sollten die angesprochenen Komposita nicht als Einzelfälle betrachtet werden, sondern die Wortbildung als gängiges fachsprachliches Prinzip und Prozess erläutert werden. Am Beispiel ,die Stadterschließung' kann visualisiert werden, dass das
Suffix ,-ung' aus einem Verb ein Nomen bilden kann sowie dass der Artikel des Kompositums aufgrund des Stammwortes, die Erschließung' ebenfalls feminin ist. Geeignete Beispiele wie ,die Besiedlung - siedeln - besiedelt - unbesiedelt - der Siedler - die Siedlung - die Siedlungsfläche' machen den Schülerinnen und Schülern transparent, dass Wörter eng semantisch verwandt sind und Bedeutung abgeleitet werden kann, insbesondere, wenn die Affixe (Präfixe und Suffixe, vgl. Karatas 2005: 94f.) und ihre Funktionsweise bereits bekannt sind. Dadurch wird der potentielle Wortschatz der Schüler/innen immens gesteigert und ihre Sprachbewusstheit (language awareness, vgl. Jeuk 2013: 122) entscheidend erhöht.

\section{Syntax}

Als weiterer Bereich der Fachsprache sollte die Syntax, also der ,Satzbau' angesprochen werden. Die Fachsprache zeichnet sich durch komplexe Satzstrukturen mit Nebensätzen in diversen hypotaktischen Beziehungen aus. Das bedeutet, dass Nebensätze mit dem Hauptsatz in einer konditionalen (Bedingung), relativen (Beschreibung), kausalen (Begründung), konzessiven (Gegensatz) oder konsekutiven (Folge) Verbindung stehen. Sichtbar wird diese Verbindung durch Konjunktionen wie, wenn', ,weil', ,so dass', obwohl' etc. und Konnektoren wie Relativpronomen. Aus diesen Sätzen können regelrecht endlose Verflechtungen erwachsen. Als Erschließungshilfe der Bedeutung könnten die Konjunktionen und Konnektoren im Sinne von ,Signalwörtern' angesprochen werden, um die Beziehung der Sätze zueinander sichtbar zu machen, damit die Schüler/innen lernen, Bedeutung zu antizipieren. Ebenso verleihen Schüler/innen ihren eigenen geschriebenen und gesprochenen Texten einen fachsprachlichen Stil, wenn sie, statt einer Aneinanderreihung von Hauptsätzen, Sätze miteinander verbinden und Beziehung herstellen. Besonders herausfordernd wird ihre Erschließung, wenn Konditionalsätze (Wenn-Dann-Aussagen) nicht durch ,wenn eingeleitet werden.

Außerdem werden Beschreibungen und Erläuterungen fachsprachlich so präzise wie möglich gebildet, so dass viele Attribute mittels Partizipien und Adjektiven eingesetzt werden (,der kleine, wildschäumende, sich abkühlende, ständig fotografierte Geysir'). Schüler/innen können vor lauter Beschreibungen das Bezugswort aus dem Blick verlieren und somit den Sinn einer Aussage nicht erfassen. An diesem Punkt ist es entscheidend, ihnen zu vermitteln, dass nicht jedes einzelne eigenschaftsbeschreibende Wort exakt verstanden werden muss, sondern, dass das Bezugswort zu identifizieren ist und die Bedeutung aus dem Kontext abgeleitet werden kann (vgl. Haß 2006: 84). 
Ein weiteres Merkmal von Fachsprache ist die häufige Verwendung von Genitiv-Konstruktionen (z.B. ,Umbruchprobleme des Ruhrgebiets', ,Stabilisierung der Bodenrente $)$, die in der Alltagssprache meist von der Ersatzform ,von' substituiert werden. Passivstrukturen sind ebenfalls häufiger in Fachsprachen vorzufinden als in der gesprochenen Alltagssprache. Eine bewusste Adressierung dieser grammatischen Phänomene im Unterricht sollte nicht isoliert und ,auf Vorrat' durchgeführt werden, sondern sich aus dem Lerngegenstand ergeben. Ist die bewusste Spracharbeit dem Inhalt bzw. Lerngegenstand des Unterrichts geschuldet, kann und sollte eine gezielte und punktuelle sprachliche Sensibilisierung erfolgen (vgl. Schroeter-Brauss 2013: 130 ff.). Dieser Leitlinie folgend, muss man sich immer wieder vor Augen führen, dass (Fach-)Sprachbildungsprozesse über einen langen Zeitraum individuell angebahnt werden und man immer nur an passenden Stellen und Momenten kleinere Bausteine adressieren kann, um Schüler/innen nicht zu überfordern.

\section{Kontinuierliche Texte (Fließtexte)}

Sachtexte in der Geographie haben meist eine deskriptive, analytische, informierende oder appellierende Funktion. Sie charakterisieren sich durch die bereits beschriebenen Merkmale der konzeptionellen Schriftsprache und weisen eine sehr hohe, sprachlich komprimierte Informationsdichte auf. Die hohe Dichte an Fachtermini, die komplexen Satzstrukturen und elaborierten, zusammenhängenden Texte überfordern einige Schüler/innen beim Lesen und Verfassen eigener Sachtexte. Ein sprachsensibler Geographieunterricht fördert daher gezielt die benötigte fachsprachliche Kompetenzanbahnung mittels Leseund Schreibstrategien/-training im Kontext einer zu erarbeitenden geographischen Fragestellung. Ziel dabei ist, die Lernenden letztlich in ihrem Wissens- und Kompetenzaufbau autonom werden zu lassen (vgl. Leisen 2013; Schmölzer-Eibinger et al. 2012, u.v. m.).

\section{Diskontinuierliche Texte}

Ein wesentliches Merkmal der geographischen Fachsprache - bzw. Kommunikation im weiteren Sinn - ist die starke Hinwendung zu diskontinuierlichen Texten. Dabei handelt es sich in Abgrenzung zu den kontinuierlichen Texten um Arbeitsmedien/-techniken unterschiedlichster Art wie Karten, Diagramme etc., die, neben den üblichen textlichen Elementen, durch eher visuelle Informationsvermittlung geprägt sind (vgl. Haubrich 2012: 20; Rinschede 2007: $107 \mathrm{f}$; DGfG 2008: 18 ff.). Die Bearbeitung der fachimmanenten, diskontinuierlichen Textsorten wie Karten,
(Klima-)Diagramme, Schaubilder, Tabellen u.v.m. benötigt systematische Kenntnis über ihren jeweiligen Aufbau, ihre Funktion und Lesart sowie spezifische sprachliche Erschließungszugänge und Redemittel (vgl. Schnotz \& Dutke 2004; Beese et al. 2013: 123).

Die geographischen Arbeitsweisen und ihre Einführung sind charakteristisch für das Schulfach (vgl. Rinschede 2007: 107ff.; DGfG 2014: 19ff.). Kein anderes Fach nutzt und adressiert diese unterschiedlichen Formen von diskontinuierlichen Texten so explizit. Während die bewusste Einführung und Dekodierung dieser Arbeitsweisen samt Aufbau und Funktionsweise seit Jahrzehnten im geographischen Schulalltag implementiert sind und somit eine fachspezifische ,Lesart' im Sinne der Methodenkompetenz angebahnt wird, erweitert sich im sprachsensiblen Geographieunterricht der Blick auf die integrierte Verschränkung mit der jeweils zu nutzenden Sprache. Dabei rücken u.a. fachspezifische Redemittel bestehend aus Termini, (Teil-)Satzstrukturen sowie die Anordnung von Textbausteinen in den Fokus, die notwendig sind, um die gewonnenen geographischen Erkenntnisse in Form von Output zu verbalisieren. Ohne diese Redemittel können die geographischen Erkenntnisse kognitiv nicht exakt erschlossen, durchdacht und sprachlich geäußert werden. Die Autorin sieht insbesondere in der Arbeit mit diskontinuierlichen Texten und der bewussten Verschränkung von fachlichen und sprachlichen Aspekten ein wesentliches Alleinstellungsmerkmal des sprachsensiblen Geographieunterrichts.

Im Umgang mit diskontinuierlichen Texten ist der Blick in den bilingualen Sachfachunterricht (auch als Content and Language Integrated Learning [kurz: CLIL] bezeichnet) zu empfehlen, da sich dieser seit Jahrzehnten mit sprachlich-inhaltlichen Fragestellungen auseinandersetzt und fruchtbare Ansätze für den deutschsprachigen Unterricht liefert (vgl. Rösch 2013: 30 f.; vgl. Hoffmann 2013: 340). Das CLIL stellt nämlich „ein integriertes Konzept [dar], in dem sowohl Sachfachinhalte als auch Sprachinhalte und -formen Aufmerksamkeit zukommt" (Dalton-Puffer 2015: 116), das bereits auf Erfahrung und z.T. empirischen Evidenzen beruht. Der Einblick in die Einführung und Semantisierung von diskontinuierlichen Textsorten im Geographieunterricht in einer fremden Arbeitssprache (vgl. Hoffmann 2015b) visualisiert, wie fachlich und sprachlich integrativ im Unterricht gearbeitet werden kann. Das heißt keinesfalls, dass bilingualer Geographieunterricht im Sinne des Content and Language Integrated Learning (CLIL) und sprachsensibler Geographieunterricht, der in der Schulsprache Deutsch durchgeführt wird, deckungsgleich sind. Die Voraussetzungen unterscheiden sich stark voneinander. Aber bewährte Prinzipien wie bspw. das Scaffolding, die verstärkte Visualisierung, 
der fachlich angeleitete Lese- und Schreibstrategieaufbau und die gezielte Wortschatzarbeit können ebenso für den deutschsprachigen Unterricht adaptiert und bei Bedarf binnendifferenzierend eingesetzt werden. Insbesondere Scaffolding kann als vorübergehendes Unterstützungssystem für die Arbeit mit geographischen diskontinuierlichen Texten im Sinne von Erschließungs- oder Verbalisierungsinstrumenten für das ,Lesen' und Schreiben fruchtbar gemacht werden. Das Prinzip lässt sich aber auf sämtliche Lerngegenstände übertragen. Aus diesem Grund wird das Prinzip des Scaffoldings im folgenden Kapitel gesondert vorgestellt.

\section{Scaffolding}

Ein wesentlicher Bestandteil des sprachsensiblen Fachunterrichts und ebenso des CLIL ist das Konzept des Scaffoldings, welches sich explizit damit auseinandersetzt, wie sich im Fachunterricht die Aneignung fachlicher Inhalte und Kompetenzen (fach-) sprachlich unterstützen lässt. Die Leitgedanken dieses Konzepts gehen auf den Psychologen Lew Wygotski (1987) zurück. Dieser beobachtete, wie Kinder in der Interaktion mit Erwachsenen lernen. Dabei differenzierte er drei Zonen bzw. Bereiche. Im ersten Bereich sind die Kinder eigenständig in der Lage, eine Handlung durchzuführen. Demgegenüber liegt der Bereich, in dem sie Aufgaben und Tätigkeiten nicht erfüllen können. Zwischen diesen beiden gegensätzlichen Bereichen identifizierte Lew Wygotski die ,Zone der nächsten Entwicklung. In dieser sind Kinder fähig, mit entsprechender Unterstützung einer kompetenten Lernpartnerin bzw. eines Lernpartners, eine spezielle Handlung durchzuführen. Das Scaffolding, vom englischen Wort ,Scaffold' [dt.: Baugerüst], stellt eine vorübergehende Hilfestellung dar, die entfernt wird, wenn das Kind in der Lage ist, die Handlung eigenständig durchzuführen. Jérôme Bruner hat dieses Prinzip für das institutionelle Lernen elaboriert, und Pauline Gibbons hat es 2002 schließlich für den Bereich des Zweitspracherwerbs weiterentwickelt (vgl. Gibbons 2002; Kniffka \& Siebert-Siebert-Ott 2012: 108 ff.; vgl. Kniffka 2010; vgl. Jeuk 2013: 138 f.).

Das Scaffolding schließt vorübergehend die Lücke zwischen dem, was Lernende momentan und potentiell schaffen können, wenn ihnen kompetentere Lernpartner/innen (Eltern, Lehrkräfte, ältere Peers etc.) Unterstützung zukommen lassen. Diese Unterstützungen können sowohl Denkanstöße, Anleitungen als auch Hilfestellungen sein. Im sprachsensiblen Kontext erhalten Lernende dabei so wenig sprachliche Unterstützung wie möglich, aber so viel wie nötig, um den Spracherwerb im fachlichen Kontext zu fördern (vgl. Schmölzer-Eibinger 2013: 35 f.; vgl. Leisen 2013). Dabei kann Scaffolding je nach Zielformulierung, anzubahnender Kompetenz und spezifischer Lerngruppe auf der Wort-, Satz- oder Textebene mit zunehmender Komplexität ansetzen. Die fachsprachlichen Scaffoldings besitzen eine dienende Funktion zur Erschließung und Verbalisierung des geographischen Sachverhalts. Fokus des Unterrichts und der planerischen Entscheidungen bleibt die Geographie!

Das Scaffolding nach Gibbons setzt sich aus dem Makro-Scaffolding inklusive Bedarfsanalyse, Lernstandsanalyse und Unterrichtsplanung sowie dem Mikro-Scaffolding (Unterrichtsinteraktion) zusammen. Dabei wird zunächst der Sprachbedarf eines Unterrichtsgegenstandes aus fachlicher Sicht geprüft und anschließend mit dem Sprachstand der Lerngruppe oder einzelner Lernender abgeglichen. Diese beiden Analysen bilden den Ausgangspunkt für die Unterrichtsplanung bezüglich Vorwissen, (Zusatz-) Material, Sequenzierung der Lernaufgabe, Sozialformen und metasprachlicher Phasen. Im Mikro-Scaffolding wird eine bewusste Fokussierung auf die Qualität der Lehrpersonen-Schüler/innen-Interaktion gelegt hinsichtlich authentischer, umfangreicher Sprachhandlungen, der Verlangsamung der Kommunikation, der Planungszeit für die Schüler/innen sowie des aktiven Zuhörens und Re-Kodierens der Schüler/innenaussagen durch die Lehrkraft (vgl. Kniffka 2010).

Im Fremdsprachenunterricht und CLIL wird das Konzept des Scaffolding schon seit Jahren eingesetzt (vgl. Thürmann 2010; vgl. Wildhage \& Otten 2008). Für das CLIL wurde es u. a. von Wolfgang Zydatiß (2010) in das Input- und Output-Scaffolding differenziert. Das Input-Scaffolding dient der Vorentlastung von Texten und Materialen, um den Erschließungs- und Verstehensprozess der Schüler/innen zu unterstützen. Verschiedene Verfahren werden dafür angeführt. Sprachlich-diskursive Verfahren geben Annotationen an, heben key words hervor und strukturieren Texte in Bezug auf Inhalt und Layout vor. Graphisch-visuelle Verfahren machen sich das Potential von Illustration durch Fotos, Piktogramme u. ä. zunutze. Ebenso können Unterrichtsmethoden Unterstützungssysteme zur Erschließung darstellen, inklusive Aufgabenstellungen zu den Bereichen pre-/ while-/post-reading or viewing, Einsatz von Lesetechniken und Wörterbucharbeit. Ferner werden Maßnahmen der Bewusstheitsbildung genutzt, die den Fokus auf bereits Verstandenes legen, statt sich auf die nicht-verstandenen Aspekte zu konzentrieren.

Das Output-Scaffolding fokussiert auf Techniken der mündlichen und schriftlichen Textproduktion, bei denen Fachwortschatz und fachkommunikative Redemittel zur Verfügung gestellt werden. Opera- 
toren stützen die Arbeitsaufträge, damit klar wird, welche Diskursfunktionen zu nutzen sind. Durch die bewusste Adressierung der geforderten Sprachhandlung (z. B. ,Beschreiben“ im Kontrast zu ,Beurteilen“) in Verbindung mit den jeweils typischen Redemitteln oder Satzmustern, wird der zu erstellende Text für Schüler/innen transparenter. Ebenso geben vorstrukturierte Lernprodukte in Form von Tabellen und Schaubildern Hilfestellung. Dem Prozess des Schreibens sollte besondere Aufmerksamkeit zuteil werden, da das Schreiben einen sehr komplexen, schwierigen Vorgang darstellt und Schüler/innen oftmals überfordert. Essentiell sind dabei eine geeignete Schreibdidaktik zur Anbahnung von Schreibprozessen. Insbesondere das bewusste Trennen der Teilbereiche Vorbereitung (Ideen generieren, ordnen etc.), Planen (Adressatenbezug, Textsorte), Schreiben (Verschriftlichen der Gedanken in einer logischen Reihenfolge) und speziell das Überarbeiten des Produktes stehen im Vordergrund und sollten kleinschrittig angeleitet werden. Hilfreich ist dabei eine Peer-to-Peer-Schreibberatung, um kooperativ Schreibkompetenzen auszubauen (vgl. Zydatiß 2010: 2 ff.).

\section{Beispielhaftes Scaffolding für die Arbeit mit der diskontinuierlichen Textsorte ,Bild'}

Das im Folgenden präsentierte Beispiel dient der Erläuterung des Prinzips von Scaffolding. Unterstützungssysteme können in unterschiedlichen Unterrichtsphasen eingesetzt und mit Blick auf das jeweilige Unterrichtsziel individuell konzipiert werden. Wesentlich ist die Passung von fachlichem und (fach-) sprachlichem Lernen.

Wie bereits erläutert, wird sprachsensibler Geographieunterricht mit Blick auf die Fachlogik und die Anbahnung fachlicher Kompetenzen und Sachverhalte geplant und durchgeführt. Die (Fach-)Sprache hat dienende Funktion, um geographische Sachverhalte präzise zu erarbeiten. Das Beispiel illustriert den Umgang mit einem Bild als diskontinuierlichen Text vor dem Hintergrund eines spezifischen geographischen Lerngegenstandes. Die Beispielmaterialien setzen sich aus Input- und Output-Scaffolding zusammen, welche die Fähigkeit der systematischen, fachlich adäquaten Bildbeschreibung in einem fach- bzw. bildungssprachlichen Register fördern sollen. Bilder oder Fotos werden in der Geographie, wie in anderen Fächern auch, sehr oft eingesetzt. In den Gesellschaftswissenschaften und besonders der Geographie dominiert jedoch nicht ihre ästhetische, sondern informierende Funktion. Die Fähigkeit zielgerichtet geographisch relevante Informationen aus ihnen abzuleiten und diese passend zu verbalisieren, steht im Fokus. Der geographische Kontext macht das Bild in diesem Fall zu einem genuin geographischen diskontinuierlichen Arbeitsmedium.

Das Scaffolding wird für folgendes unterrichtliches Szenario angedacht: Im Rahmen einer Doppelstunde innerhalb einer Unterrichtsreihe zu Naturgefahren sollen Schüler/innen der Klasse 7 die Folgen des Hurrikans Irma in der Karibik und Florida im September 2017 erläutern können. Den Schülerinnen und Schülern werden zur Erreichung dieses Ziels verschiedene Arbeitsaufträge und Materialien zur Verfügung gestellt. Zu Beginn der Unterrichtsstunde wird den Schülerinnen und Schülern ein Foto als Einstieg präsentiert. Dieses sollen sie beschreiben und Fragestellungen für die Stunde entwickeln (Bsp.: Welches Ereignis führte zu den beobachtbaren Schäden auf dem Foto? Was genau ist passiert? Welche Folgen hatte der Sturm für die betroffenen Menschen und Regionen?).

Eingebettet in den fachlichen Kontext zu den Folgen des Hurrikans, setzt die fachsprachliche Förderung in diesem Unterrichtsbeispiel bei der Bildbeschreibung an, die systematisch und strukturiert durchgeführt werden soll. Für eine präzise Beschreibung des Fotos benötigen die Schüler/innen nicht nur Wissen bezüglich der strukturierten Vorgehensweise zur Bildbeschreibung, sondern ebenso Redemittel auf Ebene von Wörtern, Satzstrukturen bis hin zum Verständnis von Beschreibungstexten. Ein mit Bildbeschreibungsinventar angereichertes Foto und eine Wortliste dienen als Input-Scaffolding der thematischen Erschließung und der sprachlichen Vorentlastung. Boxen zur Vorgehensweise stellen ebenfalls Input-Unterstützungssysteme zum Umgang mit dem diskontinuierlichen Text dar. Materialien mit den hilfreichen Wörtern und Satzstrukturen bereiten als Output-Scaffolding die Versprachlichung der relevanten Informationen vor. Vorgehensweise und Verbalisierung werden somit integriert angebahnt und den Schülerinnen und Schülern transparent vorgestellt (s. Anhang).

Die Scaffoldings lassen sich verwenden, um gezielt die fachimmanente Arbeitstechnik der Bildbeschreibung einzuführen und systematisch mit bildungssprachlichen Strukturen zu verknüpfen, die für die Erarbeitung geographischer Sachverhalte unabdingbar sind. Angelehnt ist es an die "How to Work With“Scaffolding aus dem Content and Language Integrated Learning (vgl. Hoffmann, 2015b). Lehrkräfte des deutschsprachigen Geographieunterrichts melden oft zurück, dass sie bei ihren Schülerinnen und Schülern immer wieder Defizite bei der Verbalisierung beobachten. Dabei fehlen speziell sprachliche Redemittel und strukturierte gedankliche Vorgehensweisen, die miteinander verbunden sind. Bisher geben Lehrkräfte an, kein probates Mittel gefunden zu haben, um gezielt Modifikationen zu initiieren. Gerade dieser 
Missstand soll mit dem Scaffolding adressiert werden, indem die Beschreibung eines Bildes transparent erläutert und mit passenden (fach-)sprachlichen Redemitteln verbunden wird. Es lässt sich in verschiedene Varianten mit Blick auf die jeweilige Lerngruppe weiter binnendifferenzieren (zum Beispiel durch Herausgeben eines Modelltexts).

Bei der Verwendung sollten Lehrkräfte darauf achten, dass sie den Zweck der Übung und des Arbeitsmaterials zur Anbahnung der geographischen Fachsprache strukturiert erläutern. Bei Schülerinnen und Schülern soll nicht der demotivierende Eindruck eines unreflektierten ,Malen nach Zahlen' erweckt werden, sondern eine strukturierte, systematische sprachliche Orientierungshilfe zur Verfügung gestellt werden. Die Hilfe wird nicht mehr eingesetzt, sobald die Fähigkeit, Bilder systematisch und sprachlich adäquat $\mathrm{zu}$ beschreiben, erworben wurde. Ein bewusstes $\mathrm{Ab}-$ heften von solchen fachlich und sprachlich integriert vorgehenden Materialien zum Umgang mit diskontinuierlichen Texten ist ratsam, damit Schüler/innen diese bei Bedarf als Maßnahme der Binnendifferenzierung einsetzen können - ob konkret im Unterricht oder als Vorbereitung für schriftliche Klausuren in der Oberstufe.

\section{Fazit und Schlussfolgerungen}

Intention des Artikels ist, (erste) Impulse für die Fachdidaktik und Unterrichtspraxis zu offerieren und für den Ausbau des sprachbewussten Lernens und Lehrens im Fach Geographie zu plädieren. In den kommenden Jahren gilt es das Feld des sprachsensiblen Geographieunterrichts auszuloten, die im Fach verwendete Sprache dezidiert zu erforschen und unterrichtliche Implikationen zu konzipieren, empirisch zu erproben und zu evaluieren. Daher werden zunächst einige Aspekte in diesem Beitrag vorgestellt, die ein sprachbewusstes Handeln im Geographieunterricht charakterisieren. Sie stammen aus der Theorie und Praxis der assoziierten Bereiche des bilingualen Sachfach-, modernen Fremdsprach- und Deutsch-als-Zweitspracheunterrichts. Abhängig von der jeweiligen Lernzielsetzung, der Kompetenzanbahnung, der Aufgabenkomplexität, des (Fach-)Sprachniveaus des zu erarbeitenden Lerngegenstands sowie der spezifischen Schüler/innenkohorte und ihren Bedürfnissen gilt es den Unterricht mit Blick auf Sprache zu erweitern und ggf. Scaffoldings als eine mögliche Maßnahme im sprachsensiblen Lernen anzudenken und zu entwickeln. Ziel dabei ist, Erschließungs- und Verstehensprozesse bei Schülerinnen und Schülern im fachlichen Kontext zu initiieren und mündliche und schriftliche Textproduktion bewusst vorzubereiten.
Das im vorangegangen Kapitel angedachte Scaffolding (siehe Anhang) zur Arbeit mit einem Bild innerhalb einer geographischen Fragestellung dient der Veranschaulichung, wie mit Hilfe von gezielten Unterstützungsmaßnahmen das integrierte Zusammenspiel zur Anbahnung fachlicher Kompetenzen und notwendiger Fachsprache aussehen könnte. Zugrundeliegende theoretische Überlegungen zur Sprachbildung münden in die Konzeption ein. Im Fokus eines sprachsensiblen Geographieunterrichts steht schließlich die Verschränkung fachlicher und (fach-) sprachlicher Kompetenzen. Befürchtungen, dass die Sprachbildung eine dominantere Funktion als die Anbahnung fachspezifischer Sachverhalte und Kompetenzen erhalten könnte, können abgemildert werden. Ausgangspunkt aller unterrichtlichen Entscheidungen bleibt das Fach mit dem Unterschied, dass die verwendete, notwendige Sprache und ihre gezielte Bildung berücksichtigt werden, um alle Lernenden zu inkludieren.

\section{Literatur}

Ahrenholz, B. (2010): Bildungssprache im Sachunterricht der Schule der Grundschule. In: ebd. (Hrsg.): Fachunterricht und Deutsch als Zeitsprache. Tübingen: Schneider Hohengehren. S. 15-35.

Beese, M. et al. (2015): Sprachbildung in allen Fächern. München: Klett-Langenscheid.

Beese, M. \& C. Benholz (2013): Sprachförderung im Fachunterricht. Voraussetzungen, Konzepte und empirische Befunde. In: Röhner, C. \& B. Hövelbrinks (Hrsg.): Fachbezogene Sprachförderung in Deutsch als Zweitsprache. Theoretische Konzepte und empirische Befunde zum Erwerb bildungssprachlicher Kompetenzen. Weinheim \& Basel: Beltz Juventa. S. 37-57.

Bordengeier, E. et al. (2008): Terra 2 Erdkunde Gymnasium Nordrhein-Westfalen. Stuttgart und Leipzig: Klett.

Dalton-Puffer, C. (2015): Elemente einer ,academic literacy'. Kognitive Diskursfunktionen im englischsprachigen Fachunterricht (CLIL). In: Schmölzer-Eibinger, S.\& E. Thürmann (Hrsg.): Schreiben als Medium des Lernens. Kompetenzentwicklung durch Schreiben im Fachunterricht. Münster: Waxmann. S. 13-129.

DGfG - Deutsche Gesellschaft für Geographie (Hrsg., 2014): Bildungsstandards im Fach Geographie für den Mittleren Schulabschluss - mit Aufgabenbeispielen. 8. Aufl. Bonn: Selbstverlag Deutsche Gesellschaft für Geographie (DGfG).

Gibbons, P. (2002): Scaffolding Language, Scaffolding Learning. Teaching Second Language Learners in the Mainstream Classroom. Portsmouth: Heinemann.

Grießhaber, W. (2013): Die Rolle der Sprache bei der Vermittlung fachlicher Inhalte. In: Röhner, C. \& B. 
Hövelbrinks (Hrsg.): Fachbezogene Sprachförderung in Deutsch als Zweitsprache. Theoretische Konzepte und empirische Befunde zum Erwerb bildungssprachlicher Kompetenzen. Weinheim \& Basel: Beltz Juventa. S. 58-74.

Haß, F. (2006). Fachdidaktik Englisch. Stuttgart: Klett.

Haubrich, H. (2012): Geographie unterrichten lernen. Die neue Didaktik der Geographie konkret. 2. Aufl. München: Oldenbourg.

Hoffmann, R. (2013): Geografie. In: Hallet, W. \&. F. G. Königs (Hrsg.): Handbuch Bilingualer Unterricht. Content and Language Intregrated Learning. Seelze: KlettKallmeyer. S. 338-348.

Hoffmann, R. (2015a): Bilingualer Geographieunterricht in Deutschland eine Bestandaufnahme. In: Geographie aktuell und Schule 218/37. S. 4-17.

Hoffmann, R. (2015b): Diercke Geography. For Bilingual Classes. Volume 1. Braunschweig: Westermann.

Hövelbrinks, B. (2013): Die Bedeutung der Bildungssprache für Zweitsprachlernende im naturwissenschaftlichen Anfangsunterricht. In: Röhner, C. \& B. Hövelbrinks, B. (Hrsg.): Fachbezogene Sprachförderung in Deutsch als Zweitsprache. Theoretische Konzepte und empirische Befunde zum Erwerb bildungssprachlicher Kompetenzen. Weinheim \& Basel: Beltz Juventa. S. 75-87.

Jeuk, S. (2013): Deutsch als Zweitsprache in der Schule. Grundlagen - Diagnose - Förderung. 2. Aufl. Stuttgart: Kohlhammer.

Kniffka, G. \& G. Siebert-Ott (2007): Deutsch als Zweitsprache. Lehren und Lernen. 3. Aufl. Paderborn: Schöningh.

Kniffka, G. (2010): Scaffolding. https:/www.uni-due.de/ imperia/md/content/prodaz/scaffolding.pdf(13.09.2017).

Karatas, R. (2005): Morphologie: Die Lehre von den Bausteinen der Wörter. In: Vollmer, J. (Hrsg.): Grundkurs Sprachwissenschaft. Einführung in die Sprachwissenschaft für Lehramtsstudiengänge. 5. Aufl. München: Wilhelm Fink Verlag. S. 87-98.

KMK - Kultusministerkonferenz (2013): Konzepte für den bilingualen Unterricht - Erfahrungsbericht und Vorschläge zur Weiterentwicklung. Beschluss der Kultusministerkonferenz vom 17.10.2013. https://www. kmk.org/fileadmin/Dateien/veroeffentlichungen_ beschluesse/2013/201_10_17-Konzepte-bilingualer-Unterricht.pdf (13.09.2017).

Leisen, J. (2013): Handbuch Sprachförderung im Fach. Sprachsensibler Fachunterricht in der Praxis. Grundlagenwissen, Anregungen und Beispiele für die Unterstützung von sprachschwachen Lernern und Lernern mit $\mathrm{Zu}-$ wanderungsgeschichte beim Sprechen, Lesen, Schreiben und Üben im Fach. Stuttgart: Klett.

Oleschko, S., Weinkauf, B \& S. Wiemers (2017): Praxishandbuch Sprachbildung Geographie. Sprachsensibel unterrichten - Sprache fördern. Stuttgart: Klett.

Rinschede, G. (2007): Geographiedidaktik. 3. Aufl. Paderborn: Schöningh.
Röhner, C. (2013): Einleitung: Zweisprachliche Förderung, Mehrsprachigkeit und inklusive Sprachbildung. In: Röhner, C. \& B. Hövelbrinks (Hrsg.): Fachbezogene Sprachförderung in Deutsch als Zweitsprache. Theoretische Konzepte und empirische Befunde zum Erwerb bildungssprachlicher Kompetenzen. Weinheim \& Basel: Beltz Juventa. S. 7-17.

Rösch, H. (2013): Integrative Sprachbildung im Bereich Deutsch als Zweitsprache. In: Röhner, C. \& B. Hövelbrinks (Hrsg.): Fachbezogene Sprachförderung in Deutsch als Zweitsprache. Theoretische Konzepte und empirische Befunde zum Erwerb bildungssprachlicher Kompetenzen. Weinheim \& Basel: Beltz Juventa. S. 18-36.

Schmölzer-Eibinger, S., M. Dorner, E. Langer \& M.-R. Helten-Pacher: (2012): Sprachförderung im Fachunterricht in sprachlich heterogenen Klassen. https://www.bmb. gv.at/schulen/unterricht/ba/dic_bericht_lang_24484. pdf?5te7ru (05.07.2016).

Schöneck, W. (2005): Wortbildung: Elemente und Strukturen komplexer Wörter. In: Vollmer, J. (Hrsg.): Grundkurs Sprachwissenschaft. Einführung in die Sprachwissenschaft für Lehramtsstudiengänge. 5. Aufl. München: Wilhelm Fink Verlag. S. 99-114.

Schnotz, W. \& S. Dutke (2004): Kognitionspsychologische Grundlagen der Lesekompetenz: Mehrebenenverarbeitung anhand multipler Informationsquellen. In: Schiefele, U., C. Artelt, W. Schneider \& P. Stanat (Hrsg.): Struktur, Entwicklung und Förderung von Lesekompetenz. Vertiefende Analysen im Rahmen von PISA 2000. Wiesbaden: VS Verlag für Sozialwissenschaften. S. 61-99.

Schroeter-Brauss, S. (2013): Die Bedeutung einer systematischen Sprachvermittlung für das Lernen im Fachunterricht der Sekundarstufe I. In: Köpcke, K.-M. \& A. Ziegler (Hrsg.): Schulgrammatik und Sprachunterricht im Wandel. Berlin und Boston: de Gruyter. S. 127-150.

Thürmann, E. (2010): Zur Konstruktion von Sprachgerüsten im bilingualen Sachfachunterricht. In: Doff, S. (Hrsg.): Bilingualer Sachfachunterricht in der Sekundarstufe. Eine Einführung. Tübingen: Narr. S. 137-153.

Vollmer, H. J. (2013): Integration von inhaltlichem und fachlichem Lernen. In: Hallet, W. \& F. G. Königs (Hrsg.): Handbuch Bilingualer Unterricht. Content and Language Integrated Learning. Seelze: Klett-Kallmeyer. S. $124-130$.

Wygotski, L. (1987): Ausgewählte Schriften. Band 2: Arbeiten zur psychischen Entwicklung der Persönlichkeit. Köln: Pahl-Rugenstein.

Wildhage, M. \& E. Otten (2008): Content and Language Integrated Learning. In: ebd. (Hrsg.): Praxis des bilingualen Unterrichts. Berlin: Cornelsen. S. 12-45.

Zydatiß, W. (2010): Scaffolding im Bilingualen Unterricht: Inhaltliches, konzeptuelles und sprachliches Lernen stützen und integrieren. In: Der Fremdsprachliche Unterricht: Englisch. Scaffolding im Bilingualen Unterricht 106/44. Seelze/Velber: Friedrich Verl. S. 2-6. 


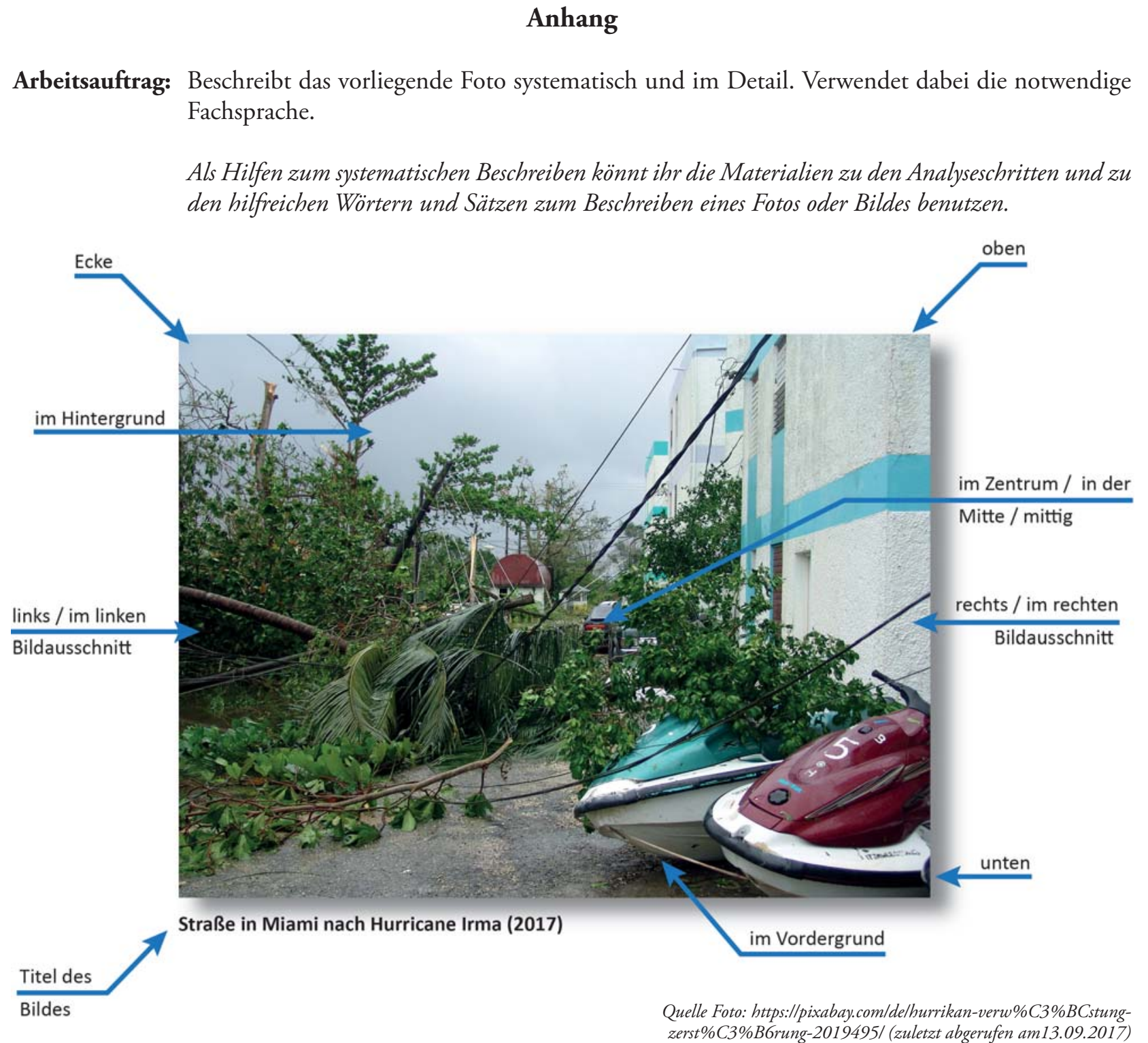

Wortliste:

die Stromleitung(en) der Jetski, die Jetskis

die Häuserzeile(n)

die Verwüstung umstürzen

der Sturmschaden, die Sturmschäden

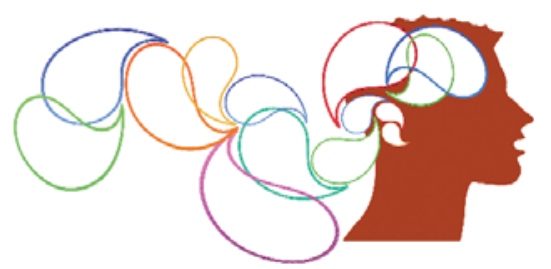

\section{Tipps, um sich einen ersten Überblick zu verschaffen}

Hilfreiche Fragen, um mehr über den Kontext eines Fotos / Bildes herauszufinden

1. Wann und wo wurde das Foto aufgenommen?

2. Welche Arten von Menschen / Aktivitäten / Landschaften werden gezeigt?

3. Wie hilft das Foto dabei, das Thema zu verstehen?

4. Wie unterscheidet sich die Situation im Foto von deiner Lebenswelt?

5. Welchen Effekt hat das Bild auf dich? 


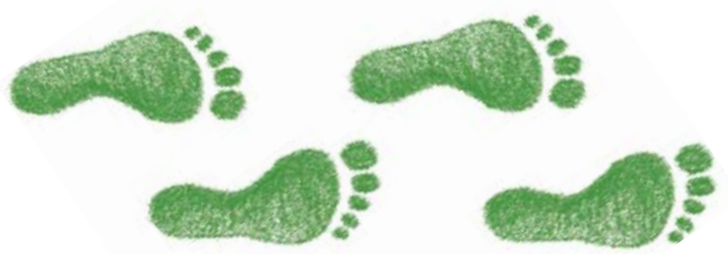

\section{Schrittfolge zur Bildbeschreibung}

Wie beschreibt man ein Bild / Foto?

1. Beschreibe das Foto systematisch: entweder ... oder ...

- von links nach rechts,

- vom Vordergrund in den Hintergrund

- vom Zentrum / Mittelpunkt nach außen

- im Uhrzeigersinn oder gegen den Uhrzeigersinn

- $\quad$ mit den wichtigsten Details beginnend.

2. Nenne die Position jedes Details, welches du ansprichst.

3. Beschreibe, was du wirklich sehen kannst.

4. Benutze viele der Beschreibungsbegriffe oder Satzteile für eine präzise Beschreibung.

5. Nutze die Fachbegriffe des Themas.

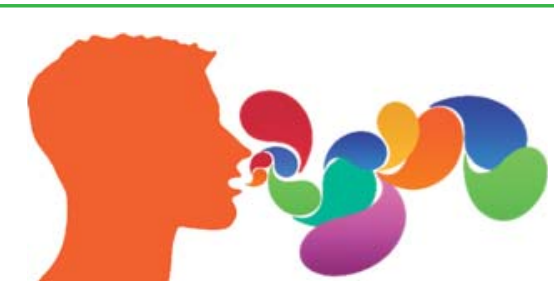

Sprache zur Bildbeschreibung

Hilfreiche Wörter und Satzteile, um eine Beschreibung von Fotos/Bildern zu beginnen:

Art des Bildes

- Das Foto ...

- Das Luftbild ...

- Das Satellitenbild ...

- Die Nahaufnahme ...

- Das Ölgemälde ...

- Der Cartoon ...

- Die Abbildung (z. B. Schaubild/Diagramm) ...

- Die Skizze ...

- u.v.m
Informationen beschreiben

- liefert Informationen über ...

- lenkt die Aufmerksamkeit auf ...

- zeigt...

- stellt ... dar.

- informiert über ...

- handelt von ...

- illustriert...

- fokussiert auf ...

- wurde aufgenommen ...

Der Titel des Bildes zeigt an, dass/verweist darauf, dass ...

\section{Hilfreiche Wörter und Satzteile, um Positionen zu beschreiben:}

Etwas ...

- kann gefunden werden

- kann identifiziert werden

- ist (rechts/links/oben/unten) lokalisiert/positioniert

- befindet sich
- oben ...

- unten ...

- $\quad$ in der Ferne ...

entfernt von ...

- am Rand ...

- $\quad$ in der Nähe von ...

- hinter ...

- vor...

- neben ...

- über ...

- ganz rechts/ganz links ...

- $\quad$ in der oberen/unteren Ecke rechts / links ...

- im Zentrum/im Mittelpunkt / in der Mitte ...

- $\quad$ im rechten/linken oberen/unteren Bildausschnitt 